\title{
Lower-Order $H_{\infty}$ Filter Design for Bilinear Systems
}

\section{with Bounded Inputs}

\author{
Edo Abraham*, and Eric C. Kerrigan, Member, IEEE
}

\begin{abstract}
We propose an optimization-based method for designing a lower-order Luenberger-type state estimator, while providing $L_{2}$ gain guarantees on the error dynamics when the estimator is used with the higher-order system. Suitable filter parameters can be computed by modelling the bilinear system as a linear differential inclusion and solving a set of bilinear matrix inequality constraints. Since these constraints are non-convex, in general, we also show that one can solve a suitably-defined semi-definite program to compute a bound on the level of sub-optimality. The design method also allows one to explicitly take account of linear parameter uncertainties in order to provide a priori robustness guarantees. The H-infinity estimator not only has lower real-time computational requirements compared to a Kalman filter, but also does not require knowledge of the noise spectrum. For a numerical example, we consider the estimation of the radiation force for a wave energy converter, where a low-order model is used to approximate the radiation dynamics.
\end{abstract}

\section{Index Terms}

Bilinear systems, H-infinity filtering, LPV, Wave energy, Observers

\section{EDICS Category: SSP-FILT}

\section{INTRODUCTION}

B ILINEAR systems have dynamics that are linear in the input and linear in the state, but not jointly linear in both. These systems arise in a variety of modelling processes, including the discretization of certain partial differential equations with boundary actions or the bilinearization of some nonlinear systems, within various application areas, e.g. plasma physics, quantum physics, biomedicine, metallurgy, energy harvesting, economics and ecology [1]. In this article, we consider estimator design for such systems where physical actuator constraints give rise to convex input constraints [2]. Motivated by this problem, we will investigate the design of estimators for bilinear systems with bounded inputs.

The literature on full-order $H_{2}$ and $H_{\infty}$ filters for linear systems is vast. The synthesis of full-order $H_{2}$ filters is a well-studied convex problem; the analysis and synthesis of $H_{2}$ filters in the presence of polytopic uncertainty is discussed in [3] and the literature therein. However, an $H_{2}$ filter's performance degrades for a general uncertainty in the model or when the spectral density of the disturbance is not perfectly known - the optimal variance in the estimation error is no longer guaranteed. In

Copyright (c) 2014 IEEE. Personal use of this material is permitted. However, permission to use this material for any other purposes must be obtained from the IEEE by sending a request to pubs-permissions@ieee.org.

Edo Abraham is with the Department of Civil and Environmental Engineering, Imperial College London, UK (e-mail: edo.abraham04@imperial.ac.uk).

Eric C. Kerrigan is with the Department of Electrical and Electronic Engineering and Department of Aeronautics, Imperial College London, UK (e-mail: e.kerrigan@imperial.ac.uk). This work was supported by the EPSRC. 
comparison, $H_{\infty}$ filters do not require statistical knowledge of the disturbance. In addition to being inherently robust to model uncertainty, the analysis and synthesis of $H_{\infty}$ filters also allows the explicit incorporation of various uncertainty types [4]-[6]. These cited works consider a system with structured and unstructured uncertainty and synthesize full-order $H_{\infty}$ filters with respect to the given uncertainty sets. This problem can be posed as a linear matrix inequality (LMI), which can be solved efficiently [7]. As we review in the following, LMI-based $H_{\infty}$ analysis and synthesis tools have been generalized to some nonlinear and bilinear systems. However, these standard methods produce estimators that have the same order as the model. For high-order systems, this may pose a high computational burden or even infeasibility for real-time implementation. In the following, we investigate the design of full-order and lower-order $H_{\infty}$ filters for bilinear systems.

The filter is based on a lower-order model of the plant, but with closed-loop robust disturbance attenuation guarantees around the full-order model. A discussion of linear parameter varying (LPV) systems and quadratic stability and performance for polytopic linear differential inclusions is presented and adapted to treat the bilinearizing inputs as linearly varying parameters; this allows the bilinear estimator dynamics to be posed as an equivalent polytopic differential inclusion. The LPV formulation is used to pose the filter design problem as an $L_{2}$ gain minimization problem for a polytopic system. The resulting optimization problem is non-convex, due to bilinear matrix inequality (BMI) constraints, hence we propose an LMI-based coordinate descent algorithm to find sub-optimal solutions from a feasible initial condition. If a solution exists, a method for finding a feasible initial observer is given for the LMI method. Since the LMI-based algorithm is a local method for a non-convex BMI optimization problem, the optimality gap to a global solution is not known. However, we show that one can compute an upper bound on the optimality gap through the solution of a full-order convex LMI problem; when this bound on the optimality gap is small, it verifies that the local solution is close in performance to a globally optimal solution. The feasibility of this design method is demonstrated using simulations with low-order filters.

This article is organised as follows. In Section II, we define the bilinear systems of interest and review existing literature on estimator design for such systems. In Section III, we formulate the bilinear system with input constraints as a linear parameter varying (LPV) system and show that the lower-order $H_{\infty}$ filter synthesis can be posed as an optimization problem with BMI constraints. Stability and quadratic performance of polytopic linear differential inclusions (PLDIs) is also reviewed in Section III. Section IV presents an LMI-based coordinate descent algorithm. We also state the dynamics for a Kalman filter for later comparison with our $H_{\infty}$ filter. In Section V, we compare the two filters by examining robust estimation problems for the wave energy converter (WEC) in [2].

\section{Problem Statement and Related Work}

We consider continuous-time bilinear systems of the form:

$$
\begin{aligned}
& \dot{x}(t)=A x(t)+\sum_{i=1}^{m} u_{i}(t) N_{i} x(t)+B u^{l}(t)+R w(t), \\
& y(t)=C x(t)+D v(t), \\
& z(t)=L x(t), \quad x(0)=x_{0},
\end{aligned}
$$

where $x(t) \in \mathbb{R}^{n}$ is the state vector, $y(t) \in \mathbb{R}^{p}$ is the measured output and $z(t) \in \mathbb{R}^{r}, r \leq n$, is the vector to be estimated. The disturbance signal $d(t):=\left[w(t)^{T} v(t)^{T}\right]^{T} \in \mathbb{R}^{q}$ is a vector containing both process noise $w(t)$ and measurement noise 
$v(t)$. The control input $u(t):=\left[u_{1}(t) \cdots u_{m}(t)\right]^{T} \in \mathbb{R}^{m}$ is known and enters the dynamics through the bilinear terms, whereas $u^{l}(t) \in \mathbb{R}^{k}$ represents known control inputs entering the dynamics linearly, hence the superscript $l$ to denote this. $A,\left\{N_{i}, i=\right.$ $1, \ldots, m\}, B, R, C, D, L$ are constant real matrices with compatible dimensions. Our objective is to design a dynamic estimator that will produce estimates of $z$ from the measurement $y$.

An important assumption we make here is that the control inputs $u(t)$ are bounded, i.e. $u(t) \in \mathbb{U}, \forall t \geq 0$, where $\mathbb{U}:=\left\{u(t) \in \mathbb{R}^{m}:\left|u_{i}(t)\right| \leq 1, \forall t \geq 0, i=1, \ldots, m\right\}$. This unit constraint is general enough to include any other component-wise bounds; if $u_{i}(t) \in\left[a_{i}, b_{i}\right], \forall t$, then substituting $\tilde{u}_{i}(t) \in[-1,1]$ for $u_{i}(t)$ such that $u_{i}:=\frac{b_{i}-a_{i}}{2} \tilde{u}_{i}+\frac{b_{i}+a_{i}}{2}$ gives a bilinear system in the standard form. The new control input $\tilde{u}(t)$ and the system matrices change appropriately, i.e. $N_{i} \leftarrow \frac{b_{i}-a_{i}}{2} N_{i}$ and $A \leftarrow A+\sum_{i=1}^{m} N_{i} \frac{b_{i}+a_{i}}{2}$.

The LMI setting has been used in full-order $H_{\infty}$ filter design for a variety of Lipschitz nonlinear systems and bilinear systems. The works in [8]-[11] make use of quadratic Lyapunov functions both to check the existence of and synthesize stabilizing Luenberger-type nonlinear estimators. Sufficient conditions for the existence of such a filter are given in [8] for a system with no disturbances. By using input-to-state stability (ISS) theory, [9] gives LMI conditions for guaranteeing asymptotic stability of the estimation error; the same LMIs are then adapted for filter design in the presence of process and measurement noise. In [10], in addition to Lipschitz nonlinearities and disturbances, linear time-varying uncertainties are also considered. An $H_{\infty}$ estimator is designed using LMI techniques; a multi-objective convex optimization problem is solved to guarantee a given error decay rate and $H_{\infty}$ disturbance attenuation level for a given Lipschitz uncertainty set. Similarly, the work in [11] considers a weighted convex minimization problem; the minimum eigenvalue of a Lyapunov matrix is maximized while minimizing the induced $L_{2}$ gain from the disturbance to the estimation error. Although these methods are applicable to full-order filter design for the bilinear case (the bilinear terms with bounds on the input are Lipschitz nonlinearities in the states), recent literature has instead pursued a less conservative approach using polytypic linear system representations for the bilinearities [12], [13].

The methods and references in [12], [13] consider what they call low-order functional $H_{\infty}$ filters. They propose a filter of the form

$$
\begin{aligned}
& \dot{\eta}=H_{0} \eta+\sum_{i=1}^{m} u_{i} H_{i} \eta+J_{0} y+\sum_{i=1}^{m} u_{i} J_{i} y+G u, \\
& \hat{z}=\eta+E y, \quad \eta(0)=\eta_{0},
\end{aligned}
$$

where the state vector of the estimator $\eta(t) \in \mathbb{R}^{r}, r \leq n$, is the same size as $z(t)$ and all the estimator system matrices in (2) are to be determined.

This formulation has some attractive properties. Firstly, the order of the estimator dynamics is $r$, which can be much less than $n$. Moreover, the Lyapunov approach is used to transform quadratic stability and $H_{\infty}$ disturbance attenuation conditions on the error system into LMIs, which are solved using efficient interior-point and semidefinite program (SDP) solvers. In addition, this framework also allows the explicit incorporation of robustness against other linear parameter uncertainties.

One of the main constraints in [12], [13] is the objective to make the filter unbiased, i.e. the dynamics of the filtering error $(e=z-\hat{z})$ is independent of the state $x$ and input $u$ of the system (1). This assumption on the filter is used to derive a number of algebraic conditions (a set of Sylvester equations) that must be solvable to guarantee the existence of such a stabilizing unbiased estimator. These equations admit a solution if and only if a given rank constraint (see [12, Eq. 15] and [13, Eq. 18]) is satisfied; this condition roughly requires that the space spanned by $\left(L A_{0}, \ldots, L A_{m}, C A_{0}, \ldots, C A_{m}, L, C\right)$ be the same as the 
one spanned by $\left(C A_{0}, \ldots, C A_{m}, L, C\right)$. This purely technical condition may not be met by many systems of the form (1); our motivating example (see Section V) does not satisfy this condition.

For systems where these nominal unbiasedness constraints are met, the observer matrices are parameterized by the solutions of the unbiasedness rank constraints, which results in error dynamics with nonlinear terms in the unknown parameters. These nonlinear terms are linearized by arbitrarily setting some nonlinear terms to zero to formulate the quadratic stability and $L_{2}$ gain attenuation conditions of the error dynamics as an LMI. In [13], by considering the control inputs as unknown but bounded uncertainties, the error dynamics are reduced to a linear system with some structured uncertainties in the $A$-matrix. In addition to the nominal case, norm bounded uncertainties on the system matrices are also considered in [13], resulting in non-convex matrix inequality problems whose relaxations are solved. In [12], only the nominal $H_{\infty}$ functional filter is considered, i.e. no model uncertainties are considered. However, the online measurable inputs are used to pose the bilinear system as an LPV system; this reduces the conservatism inherent in the formulation of [13] where the inputs are treated as bounded (but unknown) structured uncertainties. Moreover, by considering a boundedness assumption also on the derivative of the control $\dot{u}$, parameter-dependent Lyapunov matrices are used in [12] to further reduce conservativeness.

The approach taken in the this article will use a combination of ideas from the literature discussed above. As in [8], [9], [11], a reasonable structure for the estimator is to use a copy of the system dynamics plus an output feedback term - a bilinear Luenberger-type estimator. Similarly to [12], we use an LPV formulation of the bilinearities in the analysis of the error dynamics and synthesis of an estimator; this should avoid the conservatism introduced in [13]. Nonetheless, since we are considering applications with possibly arbitrarily fast control rates, an example is the case where bang-bang type control inputs are used as in [2], we do not require that the derivative of the control input to be bounded. Unlike in [12], our LPV formulation will guard against arbitrarily fast input variations by using constant Lyapunov matrices.

It can also be shown that the algebraic conditions that guarantee unbiasedness are not preserved when there is uncertainty in the model [14]; since most systems will have uncertainty in the model, the utility of this approach is limited. In our work, we do not enforce an unbiasedness condition on the error dynamics. We show in Section III that a quadratically stable estimator can be derived without the need for unbiasedness. This leads to an alternative synthesis method for lower-order $H_{\infty}$ filter design. In the next section, we review quadratic Lyapunov stability and performance and their application to LPV systems.

Notation: $P^{T}$ denotes the transpose of the matrix $P, P<0(P \leq 0)$ represents a symmetric negative definite (semidefinite) matrix.

\section{LOWER-ORDER $H_{\infty}$ FILTER FOR BILINEAR SySTEMS}

Consider the following (lower-order) Luenberger-type bilinear observer for the system of (1):

$$
\begin{aligned}
\dot{x}_{f}(t) & =\tilde{A} x_{f}(t)+\sum_{i=1}^{m} u_{i} \tilde{N}_{i} x_{f}(t)+\tilde{B} u^{l}(t)+G\left(y-\tilde{C} x_{f}\right), \\
\hat{y}(t) & =\tilde{C} x_{f}(t), \\
\hat{z}(t) & =\tilde{L} x_{f}(t), \quad x_{f}\left(t_{0}\right)=0,
\end{aligned}
$$

where $\hat{z} \in \mathbb{R}^{r}$ is the estimate of $z$. The state of the observer is $x_{f} \in \mathbb{R}^{\tilde{n}}$ and $r \leq \tilde{n} \leq n, \hat{y}(t) \in \mathbb{R}^{p}$ approximates the measured output $y$ and $u \in \mathbb{U}$ and $u^{l}$ are the control inputs as defined for (1). Here we take the low-order system matrices $(\tilde{A}, \tilde{N}, \tilde{B}, \tilde{C}, \tilde{L})$ 
as given and $G$ is the design variable to be determined in the filter synthesis. As in the dual control design problems, what is often done is to extract a low-order approximation of the system using model reduction [15], [16] or via successively finer spatial discretization of systems described by PDEs [17]. Using these low-order models, [17] designs low-order controllers with performance guarantees around the full-order model. Similarly, we base our filter design on such low-order models and with closed-loop $L_{2}$ error attenuation guarantees around the full-order model.

Note that posing all the observer system matrices $\left(\tilde{A}, \tilde{N}_{i}, \tilde{B}, \tilde{C}, \tilde{L}\right)$ as design variables results in a nonlinear matrix inequality problem with trilinear monomials. Even arbitrarily setting these nonlinear terms to zero as in [12], [13], it is not clear how to solve this problem for a general low-order model with $\tilde{n}<n$. Even for a linear autonomous system with disturbance inputs only, posing all the observer dynamics matrices as design variables results in difficult non-convex conditions that have been proven to be difficult to be reduced to an equivalent LMI by the projection lemma or congruence transformations [18].

Let $\eta(t):=\left[x(t)^{T} x_{f}(t)^{T}\right]^{T} \in \mathbb{R}^{n+\tilde{n}}$ and $d(t) \in \mathbb{R}^{q}$ denote the extended states and disturbance signals, respectively. The augmented/extended closed-loop system from inputs $u^{l}(t)$ and $d(t)$ to the estimation error $e(t):=z(t)-\hat{z}(t)$ is then given by:

$$
\begin{aligned}
\dot{\eta}(t) & =\bar{A}(t) \eta(t)+\bar{B}_{1} d(t)+\bar{B}_{2} u^{l}(t), \\
e(t) & =\bar{C} \eta(t), \quad x\left(t_{0}\right)=x_{0}, x_{f}\left(t_{0}\right)=0
\end{aligned}
$$

where

$$
\begin{aligned}
& \bar{A}(t):=\left[\begin{array}{cc}
A+\sum_{i=1}^{m} N_{i} u_{i}(t) & 0 \\
G C & \tilde{A}+\sum_{i=1}^{m} \tilde{N}_{i} u_{i}(t)-G \tilde{C}
\end{array}\right], \\
& \bar{B}_{1}:=\left[\begin{array}{cc}
R & 0 \\
0 & G D
\end{array}\right], \bar{B}_{2}:=\left[\begin{array}{l}
B \\
\tilde{B}
\end{array}\right], \bar{C}:=\left[\begin{array}{ll}
L & -\tilde{L}
\end{array}\right] .
\end{aligned}
$$

Below, we give some definitions and propositions that will be useful in the analysis and synthesis of the proposed bilinear filter. To define some useful terms, we first consider the linear differential inclusion (LDI)

$$
\begin{gathered}
\dot{x}(t)=F(t) x(t)+B_{d}(t) d(t), \quad \zeta(t)=C_{\zeta}(t) x(t), \\
x(0)=x_{0}, \quad\left(F(t), B_{d}(t), C_{\zeta}(t)\right) \in \Omega_{F} \times \Omega_{B} \times \Omega_{C},
\end{gathered}
$$

where $d(t)$ is an exogenous input, $\zeta(t)$ a linear function of the state $x(t)$ and the set $\Omega_{A} \times \Omega_{B} \times \Omega_{C}$ is a polytope.

In the case where $d(t)$ is identically zero, a sufficient condition for the asymptotic stability of the LDI (5) is the existence of a quadratic Lyapunov function.

Definition 1: [19, p. 61] For $d=0$, the system (5) is called quadratically stable if there exists a constant matrix $P=P^{T}>0$ such that

$$
F(t)^{T} P+P F(t)<0 \text { for all } F(t) \in \Omega_{F} .
$$

In order to characterise the performance of the estimator with respect to disturbances, we give the following definition as a generalisation of the $L_{2}$ gain for linear systems. We then state the bilinear $H_{\infty}$ filtering problem using this definition.

Definition 2: [20, Def. 5.1] Consider the LDI (5) as a mapping from $d$ to $\zeta$. If there exist nonnegative constants $\gamma$ and $\beta$ 
such that

$$
\int_{0}^{\infty}\|\zeta(t)\|_{2}^{2} d t \leq \int_{0}^{\infty} \gamma^{2}\|d(t)\|_{2}^{2} d t+\beta
$$

for all $d \in L_{2}[0, \infty)$ and all bounded $x_{0}$, then we say the $L_{2}$ gain is less than or equal to $\gamma$. The mapping has $L_{2}$-gain less than $\gamma$ if there exists some $\hat{\gamma} \geq 0$ such that (7) holds for $\hat{\gamma}<\gamma$. The smallest $\gamma$ for which there is a $\beta$ such that (7) holds is called the $L_{2}$ gain of the system.

Problem 1: The $H_{\infty}$ filtering problem is to design a gain $G$ such that, for the observer of the form (3) we have:

(I) error dynamics (4a) that are quadratically stable for all $u(t) \in \mathbb{U}$, when $\left[d^{T}\left(u^{l}\right)^{T}\right]^{T}=0$,

(II) given $\gamma>0$, the $L_{2}$ gain from the disturbance and control signals $\left[d^{T}\left(u^{l}\right)^{T}\right]^{T}$ to the estimation error $e$ is less than $\gamma$. In other words, we want to bound by $\gamma$ the $L_{2}$ gain from the signal $\left[d^{T}\left(u^{l}\right)^{T}\right]^{T}$ to $e$ as in Definition 2 .

Proposition 1: (The bounded-real lemma [19, p. 91]) For some $\gamma \geq 0$, if there exists a $P=P^{T}>0$, such that $\forall t \geq 0$

$$
\left[\begin{array}{cc}
F(t)^{T} P+P F(t)+C_{\zeta}(t)^{T} C_{\zeta}(t) & P B_{d}(t) \\
B_{d}(t)^{T} P & -\gamma^{2} I
\end{array}\right] \leq 0,
$$

then $\gamma$ is an upper bound for the $L_{2}$ gain for the system (5).

To solve Problem 1, we will first model the bilinear error system (4a) as an LPV system and then an equivalent PLDI. We then apply Proposition 1 to define the filter synthesis optimization problem.

\section{A. Linear parameter-varying systems}

LPV systems can be defined as a subset of systems described by the LDI of (5), where the time variations in the system matrices depend on an exogenous parameter $\delta(t)$ that is assumed to be known and available from online measurements [21]:

$$
\begin{aligned}
& \dot{x}(t)=F(\delta(t)) x(t)+B_{d}(\delta(t)) d(t), \\
& \zeta(t)=C_{\zeta}(\delta(t)) x(t), \quad x(0)=x_{0}, \quad \delta(t) \in \Delta .
\end{aligned}
$$

Here we further restrict our concern to the usual case where the system matrices in (9) can be represented by an affine function of the parameters. For example, consider the matrices $F_{0}, F_{1}, \ldots, F_{m}, F_{i} \in \mathbb{R}^{n \times n}, i=1, \ldots, m$, and $\delta(t) \in \mathbb{R}^{m}$ such that

$$
F(\delta(t))=F_{0}+\delta_{1}(t) F_{1}+\ldots+\delta_{m}(t) F_{m}, \quad \delta(t) \in \Delta
$$

where the parameters $\delta_{i}(t), i \in\{1, \ldots, m\}$ take values in some bounded intervals $\left[\underline{\delta}_{i}, \bar{\delta}_{i}\right]$, i.e.

$$
\Delta:=\left\{\left(\delta_{1}, \ldots, \delta_{m}\right) \mid \delta_{i} \in\left[\underline{\delta}_{i}, \bar{\delta}_{i}\right], i=1, \ldots, m\right\}
$$

With no restrictions on the parameters $\delta_{i}$ other than the finite upper and lower bounds, the set $\Delta$ is a bounded (convex) polytope with the set of its vertices defined (by simple vertex enumeration) as:

$$
\Delta_{0}:=\left\{\left(\delta_{1}, \ldots, \delta_{m}\right) \mid \delta_{i} \in\left\{\underline{\delta}_{i}, \bar{\delta}_{i}\right\}, i=1, \ldots, m\right\}
$$


By the Main Theorem of Polytope Theory [22, p. 356], it simply follows that $\Delta$ is equal to the convex hull of its vertices, i.e. $\Delta=\operatorname{conv}\left(\Delta_{0}\right)$.

Proposition 2: [23, Prop. 1.19] Let $f: \Delta \rightarrow \mathbb{R}$ be a convex function where $\Delta=\operatorname{conv}\left(\Delta_{0}\right) . f(\delta) \leq \mu$ for all $\delta \in \Delta$ if and only if $f(\delta) \leq \mu$ for all $\delta \in \Delta_{0}$.

For our purposes, the most useful feature of this representation is the fact that $F(\delta(t))$ and the LMIs (6) and (8) are affine (and therefore convex) functions of the parameters $\delta$. By Proposition 2, it follows that it is necessary and sufficient to check the stability (6) and performance guarantees (8) only at the vertices. In fact, the quadratic stability and performance analysis of PLDI systems in [19] make use of this property exactly. Moreover, if the system matrices $\left(F(\delta(t)), B_{d}(\delta(t)), C_{\zeta}(\delta(t))\right)$ in (9) are affine in the parameters $\delta \in \Delta$ in (11), then an equivalent PLDI representation of (9) is:

$$
\begin{aligned}
& \dot{x}(t)=F(t) x(t)+B_{d}(t) d(t), \quad \zeta(t)=C_{\zeta}(t) x(t), \\
& \left(F(t), B_{d}(t), C_{\zeta}(t)\right) \in \Omega \quad \forall t, \\
& \Omega=\operatorname{conv}\left\{(F(\delta), B(\delta), C(\delta)) \mid \delta \in \Delta_{0}, \Delta_{0} \text { as in }(12)\right\} .
\end{aligned}
$$

This follows simply from the fact that affine functions are both convex and concave and, as a result, $\Gamma\left(\operatorname{conv}\left(\Delta_{0}\right)\right)=$ $\operatorname{conv}\left(\Gamma\left(\Delta_{0}\right)\right)$ for any affine operator $\Gamma[24$, p. 67]. In the next section, we make use of the LPV (9) or its equivalent PLDI representation (13) to synthesise bilinear $H_{\infty}$ filters.

Lemma 1: If $u(t) \in \mathbb{U}, \forall t \geq 0$, then the error dynamics (4a) is equivalent to the polytopic linear differential inclusion (PLDI):

$$
\begin{aligned}
\dot{\eta}(t) & =\bar{A}(t) \eta(t)+\bar{B}_{1} d(t)+\bar{B}_{2} u^{l}(t), \\
e(t) & =\bar{C} \eta(t),
\end{aligned}
$$

where

$$
\begin{array}{r}
\bar{A}(t) \in \operatorname{conv}\left\{\hat{A}_{0}(G)+\alpha_{1} \hat{A}_{1}+\cdots\right. \\
\hat{A}_{0}(G):=\left[\begin{array}{cc}
A & 0 \\
G C & \tilde{A}-G \tilde{C}
\end{array}\right], \hat{A}_{m} \hat{A}_{i}:=\left[\begin{array}{cc}
N_{i} & 0 \\
0 & \tilde{N}_{i}
\end{array}\right] .
\end{array}
$$

Proof: Follows trivially from above by recalling that $\mathbb{U}$ is a simple component-wise bounded set.

A consequence of Lemma 1 is that the quadratic stability of (4a) is equivalent to the quadratic stability of the PLDI (14) [23], which can be easily verified using LMI solvers.

Here, we do not require the low-order model used in observer design to be stable. Unlike in standard $H_{\infty}$ filter synthesis for LPV systems, we require only that the error dynamics be stabilizable by output feedback. This is very advantageous in cases where the low-order model comes from model reduction or a coarse discretization of PDEs [17] and stability may not be preserved for some low-order models.

The following result allows us to find a solution for Problem 1.

Theorem 1: For a given $\gamma>0$, if there exists a gain $G$ and $X=X^{T}>0$ such that 


$$
\left[\begin{array}{ccc}
\bar{A}_{i}(G)^{T} X+X \bar{A}_{i}(G) & X \bar{B} & \bar{C}^{T} \\
\bar{B}^{T} X & -\gamma I & 0 \\
\bar{C} & 0 & -\gamma I
\end{array}\right]<0, \quad i=1, \ldots, 2^{m},
$$

where $\bar{A}_{i}(G)$ is the $i^{\text {th }}$ element of the $2^{m}$-tuple $\left(\hat{A}_{0}(G) \pm \hat{A}_{1} \pm \ldots \pm \hat{A}_{m}\right)$ with $\hat{A}_{0}(G), \hat{A}_{1}, \ldots, \hat{A}_{m}$ as defined in (14c), and with the matrices $\bar{B}:=\left[\bar{B}_{1} \bar{B}_{2}\right]$ with $\bar{B}_{1}, \bar{B}_{2} \bar{C}$ as given in (4c), then the observer in (3) solves Problem 1.

Proof: By Lemma 1, the bilinear error dynamics (4a) is equivalently represented by the PLDI of (14). By the strict bounded real lemma [25, p. 256], for a given G, (I) and (II) in Problem 1 are true for the PLDI of (14) if (15) is satisfied. Note that the matrix condition of (15) is another form of the one given in (8) of Proposition 1; the Schur complement lemma is used to express it as a linear function of $\gamma$ and the matrix $\bar{C}$.

Since we have a matrix inequality feasibility condition for the existence of an appropriate filter gain, we pose an optimization problem to find such a gain $G$ and minimise the bound on the $L_{2}$ gain $\gamma$ at the same time:

$$
\inf _{X, G, \gamma} \gamma \text { s.t. (15), } X=X^{T}>0, \gamma>0
$$

An important point to note is that, although the constraints in (16) are linear in the unknowns $X, G$ and $\gamma$ separately, they are not jointly linear in $X$ and $G$. If we partition $X$ according to the size of $A$ and $\tilde{A}$ as:

$$
X=:\left[\begin{array}{ll}
X_{11} & X_{12} \\
X_{12}^{T} & X_{22}
\end{array}\right],
$$

where $X_{11} \in \mathbb{R}^{n \times n}$ and $X_{22} \in \mathbb{R}^{\tilde{n} \times \tilde{n}}$, it is clear that the problem is not an LMI in the design parameters $X$ and $G$ together we have the complicating terms $X_{22} G$ and $X_{12} G$, which result in a non-convex bilinear matrix inequality (BMI).

Finally, unlike the case of $\mathrm{H}_{2}$ filters, $H_{\infty}$ filters have been shown to have inherent robustness against parametric variations in the system model. However, performance will not be guaranteed under uncertainties not explicitly considered during synthesis [5]. Bounded parametric uncertainties are easily treated by the analysis and synthesis methods given here. Parametric uncertainties of the system can be represented as linear variations of the dynamics matrix coefficients $A, N_{i}$, $B$, etc. in (1). Since, by Proposition 2, such affine variations can be represented by a PLDI (14), the filter can be made robust to such uncertainties in a straightforward manner by adding constraints to (15). Please see [23, Sec. 5.2.1] and [23, Sec. 5.2.2] for LMI-based methods on the analysis of robust stability for affine parameter-dependent systems with time-invariant and time-varying parameter uncertainties, respectively.

\section{B. Conservativeness of the PLDI representation}

It is usually claimed that design or analysis based on a PLDI representation is too conservative, since the convex hull would overbound the LPV system in question. Often, the time-varying parameters in an LPV have a finite rate of change and seeking a common Lyapunov function that stabilises a convex hull of the polytopic LPV can be conservative; such a common Lyapunov function based analysis aims to show stability for parameters that can change arbitrarily fast. As a result, a wealth of literature does now make use of parameter-dependent Lyapunov functions — the less stringent "affine quadratic stability" from [26] are, 
for example, applied in the filter design with input-rate constraints of [12]. By formulating the quadratic Lyapunov function as a function of the inputs, [12] uses bounds on both the input and its derivative to find parameter dependent Lyapunov matrices that guarantee stability of the error dynamics.

However, in our motivating wave energy problem considered in Section V-B, we mainly consider optimally controlled systems with bang-bang control arcs. When modelling the bilinear system using an LPV formulation, this amounts to parameters that switch arbitrarily fast, taking the system from one boundary to another (i.e. between vertices of the PLDI). Therefore, considering stability with a common Lyapunov function is necessary to guard against arbitrarily fast input variations; our approach in this case is, therefore, not a conservative one when compared to affine quadratic stability methods that make use of rate bounds on parameters.

It is also important to emphasise that, unlike for linear time invariant (LTI) systems, quadratic Lyapunov function-based stability and performance analyses give only sufficient conditions in the case of nonlinear systems, linear time-varying systems or linear parameter-varying systems. See [19, p. 73] and references therein for examples of PLDIs that are asymptotically stable but not quadratically stable. However, as explained in [21], even for an autonomous linear polytopic system, if the parameters vary arbitrarily fast, determining asymptotic stability using general (non-quadratic) methods is an NP-hard problem. It is also known that an LPV system with arbitrary time variations is asymptotically stable if and only if "a trajectory dependent quadratic Lyapunov function" exists [21, Thms 3-4]. Since computing general (nonquadratic) Lyapunov functions or trajectory-dependent Lyapunov functions (i.e. for all possible trajectories) is computationally infeasible, most methods adopt quadratic Lyapunov function-based analysis and synthesis [19], [21], [27]. Although only sufficient, these give us a computable set of conditions for stability in the form of LMIs.

Similarly, quadratic Lyapunov theory gives us a way to compute the exact $L_{2}$-gain performance for LTI systems (i.e. $H_{\infty}$ norm for transfer functions of LTI systems). On the other hand, only upper bounds for the $L_{2}$ gain of PLDIs, LPVs and some nonlinear systems can be derived using quadratic Lyapunov functions [19].

\section{Solving THE BMI PROBLEM (16)}

Although LMIs are convex and can be solved efficiently using SDP solvers, optimisation problems involving BMIs have been shown to be NP-hard [28]. Unfortunately, a number of control problems (and their dual observer design problems) end up with BMIs; see [29] for many classic robust and optimal control problems with BMIs and for which a convex LMI formulation has not yet been found. Although a number of schemes have been suggested for various specific structures, no method is effective at solving general BMIs. Global branch-and-bound based methods [30] have combinatorial complexity and are, therefore, computationally feasible only for very small (academic) problems. The sum-of-squares based and 'asymptotically global' solvers in [31], [32] are less demanding (the size of successive LMI relaxations grow as the square of the problem state size), but have only been shown to be useful in control problems with few complicating bilinear variables [33], [34]. Examples include static and PID controllers parameterised with up to three scalars and with $H_{\infty}$ performance guarantees [31].

The only publicly available and benchmark-tested general software for solving BMI inequality constraints is the commercial software PENBMI [35]. This software is based on an augmented Lagrangian formulation for general nonlinear semidefinite programming (PENNON [36]) specialised to BMI constrained problems. For a theoretical exposition guaranteeing convergence to locally optimal points and algorithm implementation details, see [36], [37]. Although this method fails for some large test 
cases and/or ill-conditioned problems, it has been shown [37] to return a local minimum for most of the $H_{2}$ and $H_{\infty}$ benchmark nonlinear SDP and BMI problems in Compl $_{e} i b$ : COnstraint Matrix Optimization Problem library [38]. We had attempted to use PENBMI for observer design for our wave energy problem with no success, since it struggled to solve the problem from feasible initial conditions.

In addition to using nonlinear program solvers to find local solutions using various methods, a solution is often sought by solving a convex relaxation of the BMI or by taking advantage of a structure in the specific problem. For example, a typical relaxation is to restrict the space of Lyapunov matrices so that the problem becomes amenable to a congruence transformation or a change of variables that result in convex (or LMI) problems [39]. A simple relaxation for problem (16) is to restrict the Lyapunov functions considered in (17) by assuming $X_{12}=0$. The BMI can then be changed into an LMI with a simple change of variables $Y=X_{22} G$. However, preliminary simulations showed this method can sometimes be infeasible even for the case where the optimization problem has a solution. Therefore, we seek a different alternative.

As is often done in control design problems with BMI constraints (see [34], [40] and references therein) we pursue an LMI-based coordinate descent scheme. This will allow one to solve BMI problems using an open-source LMI solver (see [41] for a list) where a BMI solver may fail or is not accessible. By fixing one of the complicating BMI variables, $G$ or $X$ in (16), we solve a set of LMIs alternatively from a feasible initial solution. These alternate LMIs have solutions in a subset of the BMI problem solutions; so, if we start from a feasible solution, this method remains feasible and is monotonically non-increasing. As detailed in [34], optimality of the alternating LMIs does not imply optimality in both variables — the method can get stuck before reaching a local minima. However, in practice locally optimal solutions are reached if each coordinate descent leads to a decrease in the objective function [42].

\section{A. LMI-based algorithm and initial robustly feasible observer}

From (14) and the assumption that the full-order model is quadratically stable, it can be shown that the estimation error dynamics is quadratically stable if $G$ stabilizes the lower-order $2^{m}$-tuple PLDI $\left(\tilde{A} \pm \tilde{N}_{1} \pm \ldots \pm \tilde{N}_{m}\right), i=1, \ldots, m$. Let $\tilde{F}_{i}$ be the $i^{t h}$ component of the $2^{m}$-tuple $\left(\tilde{A} \pm \tilde{N}_{1} \pm \ldots \pm \tilde{N}_{m}\right)$. A feasible filter gain $G$ can be computed by solving the convex LMI feasibility problem

$$
\left(\tilde{F}_{i}-G \tilde{C}\right)^{T} P+P\left(\tilde{F}_{i}-G \tilde{C}\right)<0, \quad i=1, \ldots, 2^{m}
$$

Although this filter may not have optimal performance around the full-order model, it will be quadratically stabilising for the error dynamics (4a) and therefore would suffice as an initialization for Algorithm 1. Using the solution as an initial condition for $G$, we can alternately solve LMIs until the noise attenuation bound $\gamma^{(k)}$ no longer improves. It is important to note here that we are not discussing stability of a closed-loop control system, but merely stability of the error dynamics as an input-output block.

A limitation of our proposed method is the computational complexity in solving (16). The number of LMI constraints grows exponentially with the number of bilinear inputs, since the number of vertices describing the PLDI is $2^{m}$, where $m$ is the number of bilinearizing inputs. Nonetheless, the LMIs are solved off-line in filter design. Therefore, for many applications where the number of bilinear inputs is small, such a design is feasible on a modern desktop computer. 


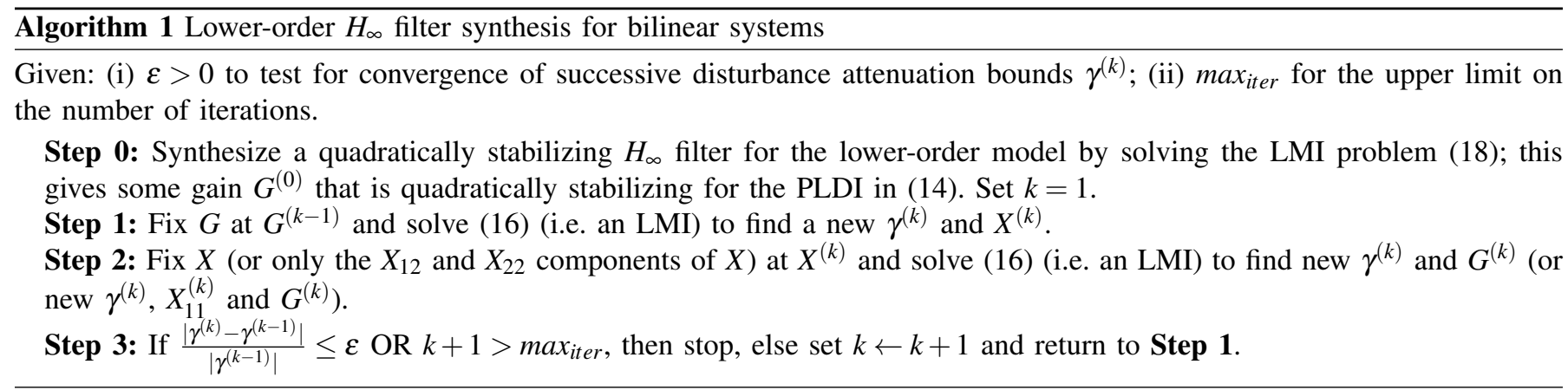

\section{B. An upper bound on the optimality gap for Algorithm 1}

A very desirable property for an asymptotically global method or solutions via convex relaxations is being able to give a certificate of global optimality or the gap of global suboptimality [32]. The method of Algorithm 1 is only a local method and so the globally optimal $L_{2}$ gain bound $\gamma_{\tilde{n}}^{*}$ is unknown for the $\tilde{n}^{\text {th }}$ order filter, where $\tilde{n}<n$. However, a full-order filter (i.e. a filter with $\tilde{n}=n$ based on the full-order model) can be expected to give the the best performing filter around the full-order model. Luckily, the full-order $H_{\infty}$ synthesis problem is a convex problem and the full-order filter can be computed by solving an SDP.

We consider the same Luenberger-type bilinear observer as before, but a full-order one:

$$
\begin{aligned}
& \dot{\hat{x}}(t)=A \hat{x}(t)+\sum_{i=1}^{m} N_{i} \hat{x}(t) u_{i}(t)+B u^{l}(t)+G(y(t)-C \hat{x}(t)), \\
& \hat{z}(t)=L \hat{x}(t), \quad x\left(t_{0}\right)=x_{0},
\end{aligned}
$$

where $\hat{x}(t) \in \mathbb{R}^{n}$ and $u(t) \in \mathbb{U}$.

In another form, substituting for $y(t)=C x(t)+D v(t)$, (19) becomes

$$
\begin{aligned}
& \dot{\hat{x}}(t)=A \hat{x}+\sum_{i=1}^{m} N_{i} \hat{x} u_{i}+G C x-G C \hat{x}+B u^{l}+G D v, \\
& \hat{z}(t)=L \hat{x}(t), \quad x\left(t_{0}\right)=x_{0}
\end{aligned}
$$

where $\hat{x}(t) \in \mathbb{R}^{n}$ and $u(t) \in \mathbb{U}$.

Let $\xi(t):=x(t)-\hat{x}(t)$ and $e(t):=z(t)-\hat{z}(t)$, then the error dynamics can be written as:

$$
\begin{aligned}
& \dot{\xi}(t)=\left(A+\sum_{i=1}^{m} N_{i} u_{i}(t)-G C\right) \xi(t)+\left[\begin{array}{ll}
R & -G D
\end{array}\right]\left[\begin{array}{l}
w(t) \\
v(t)
\end{array}\right], \\
& e(t)=L \xi(t), \quad \xi\left(t_{0}\right)=\xi_{0} .
\end{aligned}
$$

The design objective is to find $G$ such that $A+\sum_{i=1}^{m} N_{i} u_{i}(t)-G C$ is quadratically stable $\forall u(t) \in \mathbb{U}$ and the $L_{2}$ gain from $[w v]^{T}$ to $e$ is minimized. Writing the PLDI that includes (21) as before, we can use the following result:

Proposition 3: For a given $\gamma>0$, if there exists a gain $Y$ and $X=X^{T}>0$ such that for all $i \in\left\{1, \ldots, 2^{m}\right\}$

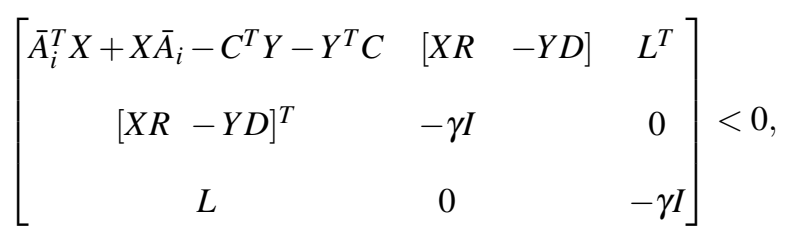


where $\quad \bar{A}_{i}$ is the $i^{\text {th }}$ element of the $2^{m}$-tuple $\left(A \pm N_{1} \pm \ldots \pm N_{m}\right)$ and $\left(A, N_{1}, \ldots, N_{m}\right), R, D, L$ are as in (21), then the observer in (19) has quadratically stable error dynamics (21) and the $L_{2}$ gain from $\left[w^{T} v^{T}\right]^{T}$ to $e$ is less than $\gamma$.

Proof: The proof follows from the strict bounded real lemma [25, p. 256] if the substitution $Y=X G$ is made. Here, $X=X^{T} \in \mathbb{R}^{n \times n}$ and $Y \in \mathbb{R}^{n \times p}$.

For the full-order $H_{\infty}$ filter above, an LMI feasibility problem is derived using a change of variables. This resulting problem is convex, since it is linear in all the unknowns $X, Y$ and $\gamma$. Minimizing this linear problem with a convex LMI constraint will give us the lowest achievable quadratic $L_{2}$-gain performance for the PLDI; because the Lyapunov matrix $X$ is positive definite, the gain can be derived from the inverse relation $G=X^{-1} Y$.

Let the optimal $L_{2}$ gain of the full-order filter be $\gamma_{n}^{*}$, with $n$ denoting the fact that the filter is full-order. Similarly, let $\gamma_{\tilde{n}}^{(k)}$ represent the gain of of the order $\tilde{n}$ filter from the $k^{\text {th }}$ iterate of Algorithm 1, and let the global solution of the optimization problem in (16) be denoted by $\gamma_{\tilde{n}}^{*}$. It then follows that

$$
\gamma_{\tilde{n}}^{(k)} \geq \gamma_{\tilde{n}}^{*} \geq \gamma_{n}^{*}
$$

and that $\gamma_{n}^{*}$ can be computed exactly by solving an SDP.

The relation (23) can, therefore, be used to upper bound the distance between the performance of the best $\tilde{n}$ order filter from (16) and its approximate solution via Algorithm 1. We have

$$
\gamma_{\tilde{n}}^{(k)}-\gamma_{\tilde{n}}^{*} \leq \gamma_{\tilde{n}}^{(k)}-\gamma_{n}^{*}
$$

If the relative optimality gap bound $\frac{\gamma_{\tilde{n}}^{(k)}-\gamma_{n}^{*}}{\gamma_{n}^{*}}$ is small, the solution from Algorithm 1 will be close, in performance, to the optimal order $\tilde{n}$ bilinear model-based $H_{\infty}$ filter.

\section{Linear Time-Varying Kalman and $H_{\infty}$ Filtering}

The linear Kalman filter (KF) is by far the most widely and successfully used filter for state and parameter estimation of linear systems [43]. With the control inputs available from real-time measurements, the bilinear system can be considered as a linear time-varying (LTV) system. As such, the LTV Kalman filter can be used. Given the system dynamics in (1), the LTV Kalman filter is then implemented as [44, 5.3.8-5.3.11]:

$$
\begin{aligned}
& \dot{\hat{x}}(t)=F(t) \hat{x}(t)+B u^{l}(t)+K(t)[y(t)-C \hat{x}(t)], \\
& K(t)=P(t) C(t)^{T} \hat{Q}_{v}^{-1}, \\
& \dot{P}(t)=F(t) P(t)+P(t) F(t)^{T}-K(t) C(t) P(t)+\hat{Q}_{w}, \\
& \hat{z}(t) \quad=L \hat{x}(t), \quad \hat{x}(0)=\hat{x}_{0}, \quad P(0)=P_{0},
\end{aligned}
$$

where $F(t):=A+\sum_{i=1}^{m} N_{i} u_{i}(t), \hat{Q}_{w}:=R Q_{w} R^{T}, \hat{Q}_{v}:=D Q_{v} D^{T}$ and $P(t) \in \mathbb{R}^{n \times n}$ is the covariance of the state estimation error. Note that we need to solve an ODE of order $n^{2}$ to find $P(t)$. The system and measurement disturbances $w$ and $v$ in (1) are assumed to be white noise with a given covariance; i.e. $w \sim N\left(0, Q_{w}\right)$ and $v \sim N\left(0, Q_{v}\right)$. A lower-order Kalman filter can be implemented as in (25), based on a lower-order model of the system dynamics (1). 
A widely used filter for state and parameter estimation of nonlinear systems is the Extend Kalman filter (EKF) [43]. As such, it is an appropriate candidate to be applied to the nonlinear system of interest in this paper. However, for a bilinear system with known inputs $u$ and $u^{l}$, as is the case here, the LTV Kalman filter is mathematically equivalent to an EKF. It simply suffices to compare (25) with the dynamics of an EKF [43, pp. 401].

Similarly to the Kalman filter, an LTV $H_{\infty}$ filter can be used. However, we do not pursue this here for a few reasons. Firstly, our aim is to design a low-order filter with stability and performance guarantees on the full-order model. The usual LTV $H_{\infty}$ filter [45] designed on the low-order model does not give performance guarantees around the full-order model. In addition to a smaller online computational cost, this guarantee is another advantage of our LPV formulation. Secondly, an appropriate noise attenuation bound $\gamma$ would have to be chosen a priori for the Riccati differential equations of the LTV $H_{\infty}$ filter [45, Sec. III]. In addition to avoiding the difficulty in choosing an appropriate $\gamma$ value a priori, our formulation allows us to incorporate robustness against parametric uncertainty in a straightforward manner; it is not clear how this would be done for the LTV case.

\section{EXAmPLE Simulations}

\section{A. Example 1}

We first consider a simple example problem to illustrate the bilinear $H_{\infty}$ filter synthesis method developed in this article. As also stated in Section II, the results in [12] and [13] cannot be applied here because the example system, similarly to the wave energy system in Section V-B below, does not satisfy the rank constraint of [12, Eq. 15] and [13, Eq. 18]. For this example, the bilinear system matrices in (1) are:

$$
\begin{aligned}
A & =\left[\begin{array}{rrrr}
-0.50 & 0.45 & 0.77 & 0.83 \\
0 & -0.45 & 0.92 & -0.93 \\
0 & -0.38 & -0.31 & -0.89 \\
0 & -0.23 & 0.75 & -0.91
\end{array}\right], \\
N & =\left[\begin{array}{cccc}
0.20 & 0 & 0 & 0 \\
0 & 0 & 0 & 0 \\
0 & 0 & 0 & 0 \\
0 & 0 & 0 & 0
\end{array}\right], \quad B=\left[\begin{array}{c}
0 \\
0.83 \\
0.80 \\
0.95
\end{array}\right] \\
R & =\left[\begin{array}{ccc}
0.80 & 0 & 0 \\
0 & 0.90 & 0 \\
0 & 0 & 1 \\
0 & 0 & 0
\end{array}\right], \quad D=1 \\
C & =\left[\begin{array}{llll}
0 & 0.49 & 0.90 & 0.13
\end{array}\right], \\
L & =\left[\begin{array}{llll}
1 & 0 & 0 & 0
\end{array}\right] .
\end{aligned}
$$




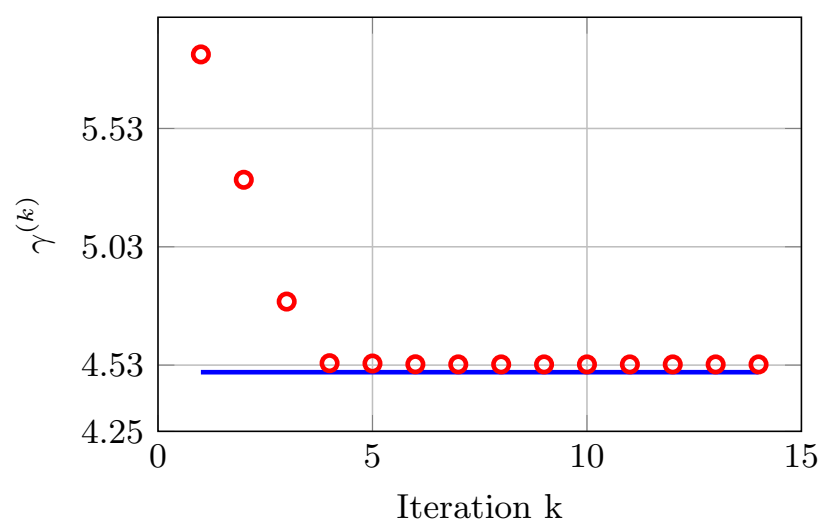

Fig. 1. Convergence of $\gamma^{(k)}$ in Algorithm 1. The lower (blue) line shows the best full-order filter performance as a bound for $\gamma^{(k)}$.

An approximate $2^{\text {nd }}$-order model of the above system is given by:

$$
\begin{aligned}
& \tilde{A}=\left[\begin{array}{cc}
-0.50 & 0.98 \\
0 & -1.34
\end{array}\right], \\
& \tilde{N}=\left[\begin{array}{cc}
0.20 & 0 \\
0 & 0
\end{array}\right], \quad \tilde{B}=\left[\begin{array}{c}
0 \\
1.87
\end{array}\right], \\
& \tilde{C}=\left[\begin{array}{ll}
0 & 0.66
\end{array}\right], \quad \tilde{L}=\left[\begin{array}{ll}
1 & 0
\end{array}\right] .
\end{aligned}
$$

The filter synthesised using Algorithm 1 gives $G=\left[\begin{array}{ll}0.2811 & 0.4587\end{array}\right]^{T}$. All computations were performed on a $2.4 \mathrm{GHz}$ Intel Core 2 Duo CPU machine in MATLAB using YALMIP [41] and SDPT3 [46]. The closed-loop disturbance attenuation bound $\gamma$ from Algorithm 1 is shown in Figure 1. The optimality gap is bounded by $0.04=4.53-4.49$.

The systems were simulated with initial conditions $x(0)=0$ and $\hat{x}(0)=[0.4820 .954]^{T}$. The inputs $u(t)=\sin (0.1257 t+1)$, $u^{l}(t)=\sin (0.0628 t+0.3)$ and $w(t)=0$ were used. The measurement noise $v$ was generated from a normal distribution and scaled so that the energy in $v$ was approximately $25 \%$ of the energy in the measurement $y$. The covariance of the state estimation error was set to $P(0)=0$ and the exact covariance of the measurement noise was used in simulating the Kalman filter, i.e. $Q_{w}=0$, and $Q_{v}=\operatorname{cov}(v)$. Figures 2 and 3 show the measurement and input, and estimated signals, respectively. The $H_{\infty}$ filter estimate converges at a similar rate compared to the Kalman filter estimates; however, the $H_{\infty}$ filter has smaller estimation errors than the Kalman filter. Similar qualitative results were observed for many other randomly generated initial conditions. Moreover, whereas the real-time implementation of the Kalman filter requires the solution of $\tilde{n}^{2}+\tilde{n}$ ODEs, the low-order $H_{\infty}$ observer solves $\tilde{n}$ ODEs. In addition, the $H_{\infty}$ estimator does not require knowledge of the statistics of the noise signals.

\section{B. Example 2}

We consider the heaving point absorber WEC from [2] where a power take-off mechanism with controlled damping and active force elements is used. A state space representation of the system was derived where the kernel representing forces due to radiated waves is approximated arbitrarily well by a finite-dimensional state-space model of sufficient order. An optimal control problem was posed and solved in [2] to determine a real-time optimization based control to be used in a receding horizon manner. The active and passive power take-off forces are controlled proportionally. It was shown that, with an identified 


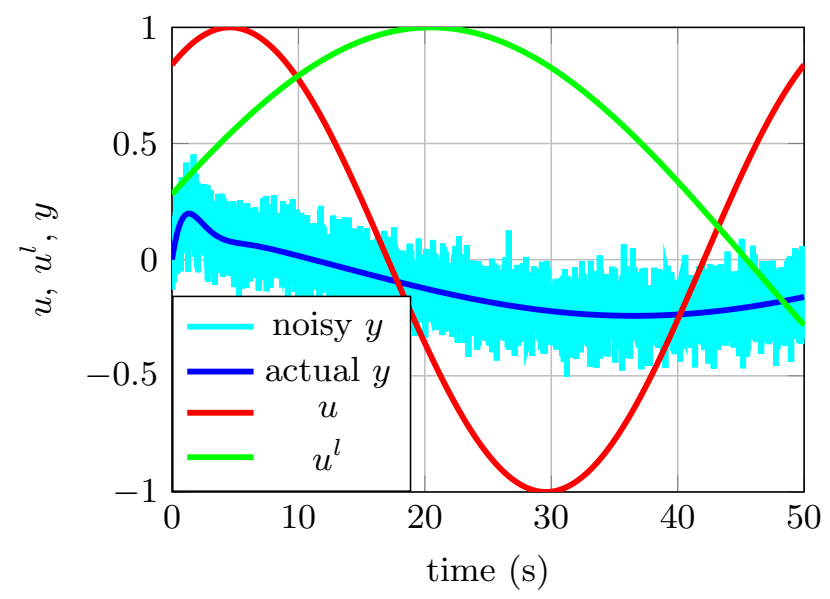

Fig. 2. Noisy measurement and control inputs

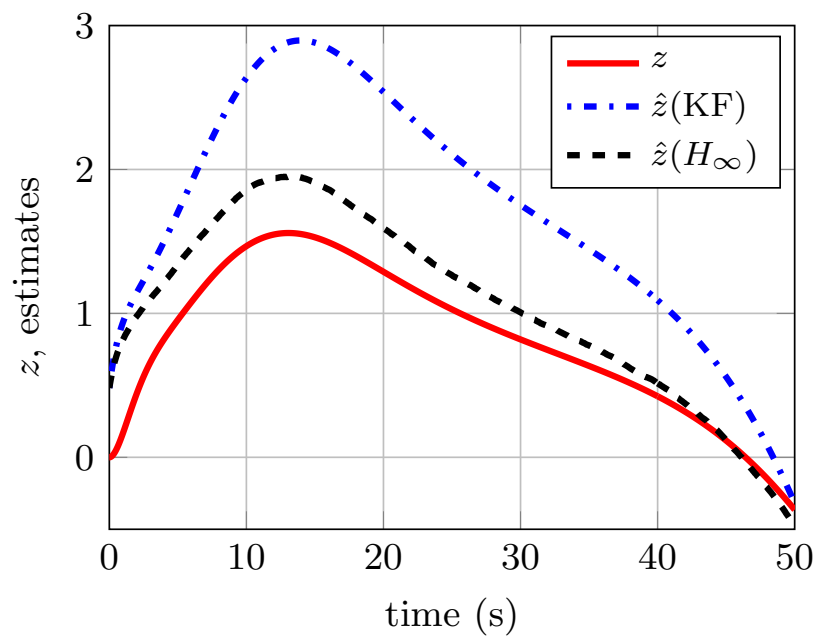

Fig. 3. Comparison of estimates from the Kalman Filter (KF) and $H_{\infty}$ filter.

radiation subsystem of order $n-2$, the complete WEC system dynamics can be re-written in state space form as:

$$
\begin{aligned}
& \dot{x}_{1}(t)=x_{2}, \\
& \dot{x}_{2}(t)=\frac{1}{M+\mu_{\infty}}\left[f_{\text {exc }}(t)+G u^{l}(t)-\right. \\
&\left.B_{p t o} u(t) x_{2}(t)-C_{r} x_{3: n}(t)-k x_{1}(t)\right], \\
& \dot{x}_{3: n}(t) \quad=A_{r} x_{3: n}+B_{r} x_{2}(t),
\end{aligned}
$$

where the $u^{l}(t) \in[-1,1]$ represents the active force and $u(t) \in[0,1]$ controls the damper force.

The variables $x_{1}$ and $x_{2}$ represent the displacement and velocity of the WEC, respectively, $f_{\text {exc }}$ is the wave excitation force and $k$ represents the restoring force stiffness. The system (28) is bilinear and of the form considered in (1); it has a bilinear term in the form of $u(t) x_{2}(t)$ with bounded $u$.

As in all the wave energy literature, the radiation force $f_{r}(t) \approx C_{r} x_{3: n}(t)$ was assumed known in the dynamic optimal control algorithm of [2]; this is not true in practice, since we cannot measure this force directly. The measurements of the velocity used in determining the optimal control inputs will also have errors in practice. For the model considered in the example simulations of [2], sufficiently high order models can approximate the radiation kernel well enough in simulation. Here, we take a $7^{\text {th }}$ order system model (i.e. $n=7$ and the radiation subsystem is of order 5) as the full-order model and synthesise a robust lower-order observer based on a $3^{\text {rd }}$ order radiation subsystem model $(\tilde{n}=5)$. All the parameters considered are as 


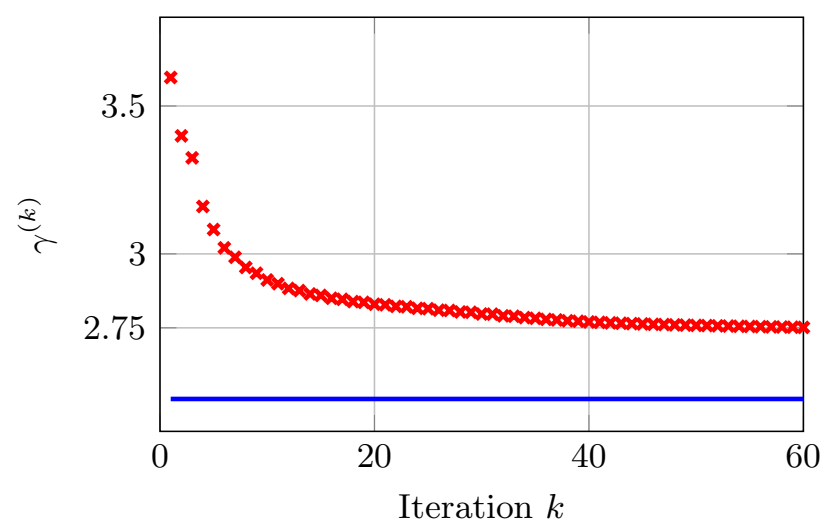

Fig. 4. Convergence of $\gamma^{(k)}$ in synthesis via Algorithm 1. The lower (blue) line shows the best full-order filter performance as a bound for $\gamma^{(k)}$.

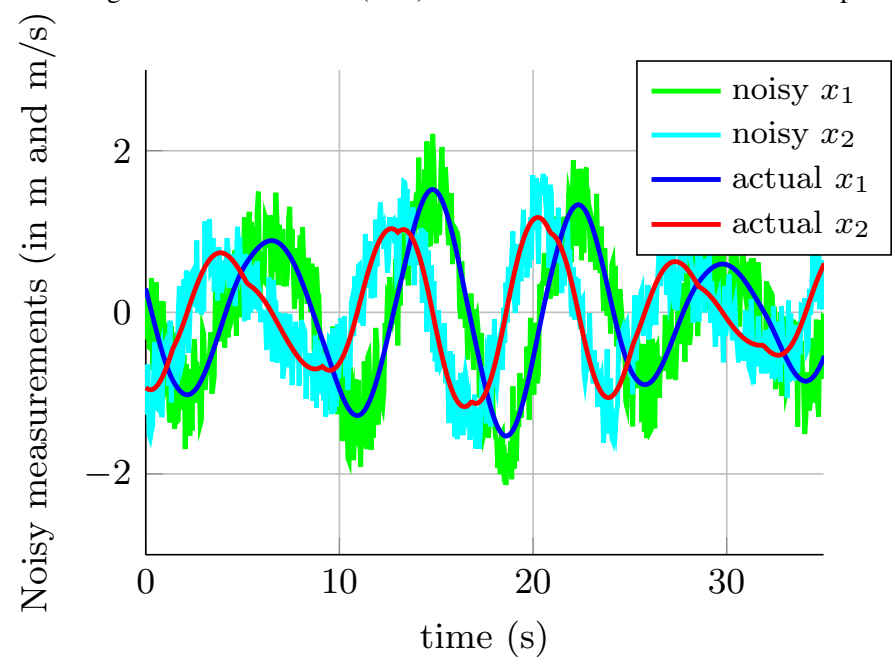

Fig. 5. Displacement $\left(x_{1}\right)$ and velocity $\left(x_{2}\right)$ and their noisy measurements

in [2, Figure 3c]. In addition, we take a nominal value of $B_{p t o}=304 \mathrm{kNs} / \mathrm{m}$. A low-order Kalman filter is constructed based on this nominal model.

For the $H_{\infty}$ filter synthesis, we take it to be the case that the parameter $B_{\text {pto }}$ is known with some uncertainty. Here, a low-order $H_{\infty}$ filter is constructed considering a parameter uncertainty of up to $\pm 30 \mathrm{kNs} / \mathrm{m}$, i.e. the parametric uncertainty $B_{\text {pto }} \in[274,334] \mathrm{kNs} / \mathrm{m}$ is explicitly included in the PLDI model used in (14). The performances of the Kalman filter and $H_{\infty}$ filters are compared under this uncertainty; the true system has $B_{p t o}=275 \mathrm{kNs} / \mathrm{m}$ in simulation. We assume that noisy velocity and displacement measurements are available. The objective is to attenuate the $L_{2}$ gain from the control inputs, excitation force and disturbances to the estimation error. A normally distributed random noise with a bound equal to the mean of the absolute actual displacement was added to the measured signals; in (1), we set $R=0.1 I_{n}$ and $D=0.1 I_{p}$. The system matrices are too large to display here.

The high order model simulations for the WEC are generated by the optimal bang-bang controller as shown in [2, Figure $3 c]$. The convergence of the closed-loop disturbance attenuation bound $\gamma$ from Algorithm 1 is shown in Figure 4 . Figure 5 shows noisy measurements of the displacement and velocity. In Figures $6 \mathrm{a}$ and $6 \mathrm{~b}$ we show snippets of the estimates and estimation errors, respectively.

Compared to the low-order Kalman filter, the low-order model based $H_{\infty}$ filter estimates seem to converge faster and with lower relative errors. The errors also depend on the initial state and covariance estimates for the KF. Figure 7 shows how the filters performances degrade for different non-zero initial state estimates. For a vector $\zeta \in \mathbb{R}^{N}$, let the $l_{p}, p=1,2, \infty$ norm be 


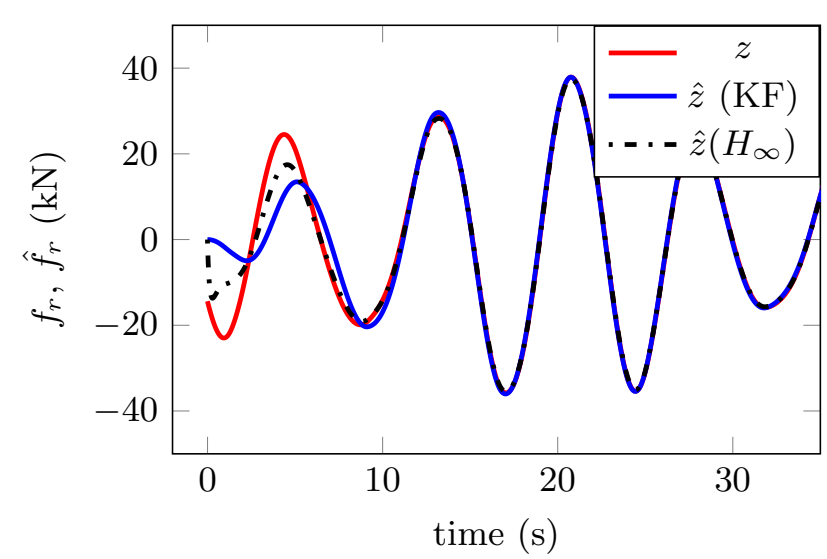

(a) estimates of the radiation force

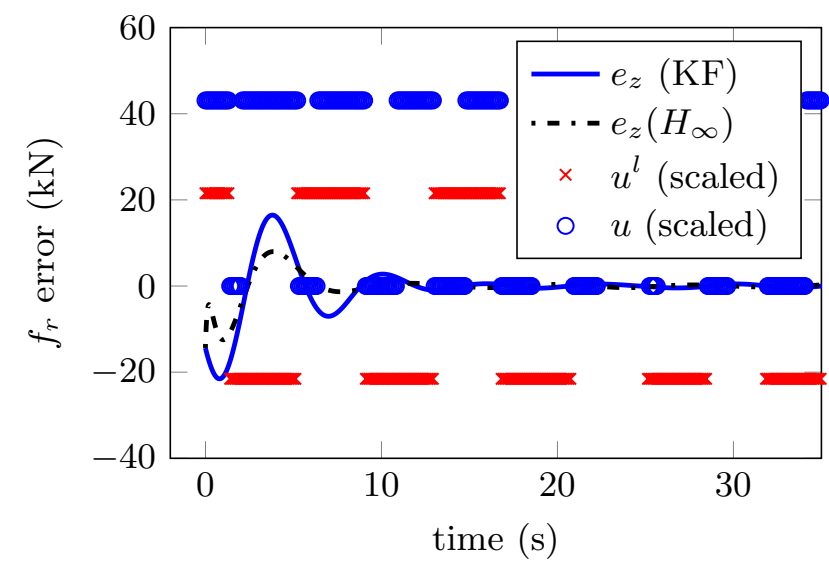

(b) errors in estimates, and scaled control inputs

Fig. 6. Comparison of radiation force estimates by the Kalman Filter (KF) and $H_{\infty}$ filter. Assuming the states are not known, initial conditions $P(0)=0$ and $\hat{x}(0)=0$ were used.

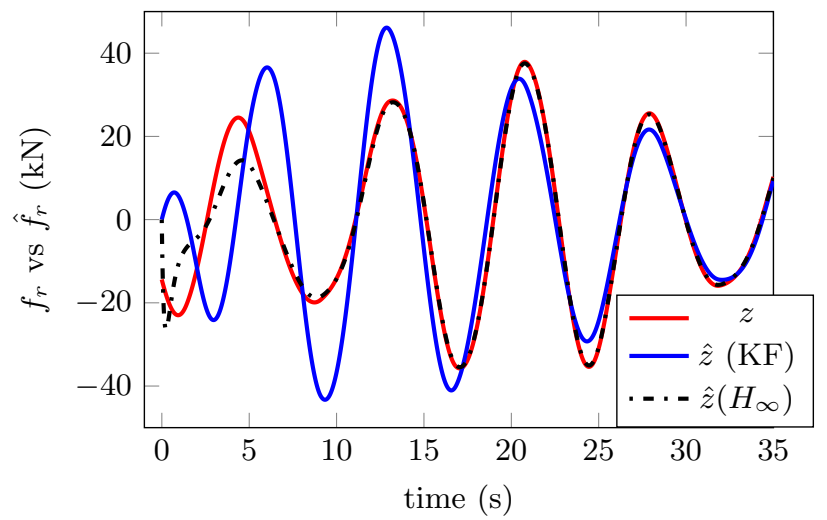

Fig. 7. Estimates of radiation force with a different initial state guess; $P(0)=0$, and $\hat{x}(0)=\left[\begin{array}{lllll}1 & -1 & 0 & 0 & 0\end{array}\right]^{T}$

defined as $\|\zeta\|_{p}:=\left(\sum_{i=1}^{N}\left|x_{i}^{p}\right|\right)^{1 / p}, p=1,2$, and $\|\zeta\|_{\infty}:=\max _{1 \leq i \leq N}\left|\zeta_{i}\right|$. The relative $l_{p}$ norm error is then defined as the ratio of the $l_{p}$ norm of the error in the estimate to the $l_{p}$ norm of the actual signal. In Table I, we present the $l_{p}$ norm of the errors and the actual signals of the simulations in Figure 6 and Figure 7. These relative norm errors are a function of how many samples we take; as the period over which estimation is performed increases, these relative errors converge to some value. As more estimation data is derived with increasing simulation time, the relative norm of the error signal is recomputed and stored. A plot of the running ratio of the $l_{1}$ norm of the error to the $l_{1}$ norm of the actual signal at different time points is shown in Figure 8. As we run forward in time, more data is used and so the errors converge towards some steady-state value. The simulation in Figure 8 is run over 35 seconds with sampling time of $0.01 \mathrm{~s}$. It is apparent that changing the initial filter state from zero in the simulation of Figure 6 to $\hat{x}=\left[\begin{array}{lllll}1 & -1 & 0 & 0 & 0\end{array}\right]^{T}$ in the simulation of Figure 7 is shown to increase the relative error for the KF more significantly than the $H_{\infty}$ filter. A similar performance was confirmed by taking more than 100 additional simulations from many random initial state estimates and the same levels of process noise.

Considering both filtering methods, it was also observed that the estimates deteriorate as the order of the model used in the synthesis decreases. This is not surprising, since a lower-order model is likely to capture less of the system dynamics. In Figures 9 and 10, we can see estimates using measurement data of velocity and displacement from a receding horizon simulation.

From these and other simulations, it appears that under some initial conditions and when the velocity is high, the $1^{\text {st }}$-order $H_{\infty}$ observer and the $3^{\text {rd }}$-order Kalman filter poorly estimate the radiation force, compared to a $3^{\text {rd }}$-order $H_{\infty}$ observer. The 
TABLE I

THE NORMS OF THE TRUE SIGNAL $f_{r}$ AND THE ESTIMATION ERRORS $\left(\hat{f}_{r}-f_{r}\right)$ FROM FIGURE 6 AND FIGURE 7.

\begin{tabular}{|l|l|l|l|}
\hline \multirow{2}{*}{ Estimation error } & \multicolumn{3}{|l|}{ norms of signals $\left(\hat{f}_{r}-f_{r}\right)$ or $f_{r}$} \\
\cline { 2 - 4 } & $l_{1}$ norm & $l_{2}$ norm & $l_{\infty}$ norm \\
\hline$H_{\infty}$, Fig. 6 & $2.37 \times 10^{3}$ & $1.24 \times 10^{2}$ & 14.4 \\
\hline$H_{\infty}$, Fig. 7 & $2.89 \times 10^{3}$ & $1.51 \times 10^{2}$ & 14.4 \\
\hline KF, Fig. 6 & $4.87 \times 10^{3}$ & $2.43 \times 10^{2}$ & 21.5 \\
\hline KF, Fig. 7 & $1.79 \times 10^{4}$ & $5.92 \times 10^{2}$ & 37.1 \\
\hline$f_{r}$ & $3.01 \times 10^{4}$ & $8.28 \times 10^{2}$ & 37.9 \\
\hline
\end{tabular}

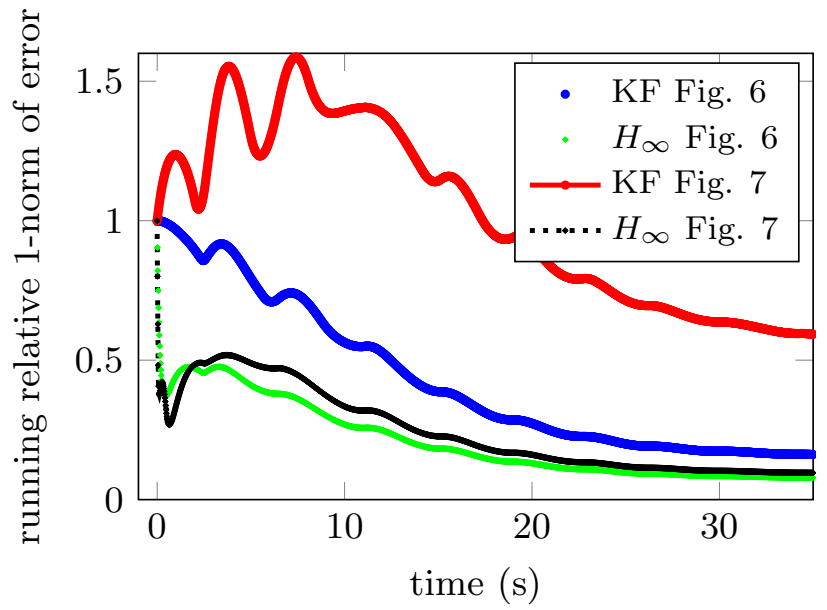

Fig. 8. Running $L_{1}$-norm relative error against time for the simulations of Figure 6 and Figure 7.

latter is also poorer at estimating the velocity. From these simulations it can be seen that a $1^{\text {st }}$-order radiation subsystem gives a filter with unsatisfactory $L_{2}$-gain bounds for the error dynamics and that the error reduces much slower compared to a $3^{\text {rd }}$-order radiation subsystem based-model.

\section{CONCLUSIONS}

In this article, the low-order $H_{\infty}$ filter design for bilinear systems with bounded inputs has been investigated. Since the robust unbiased functional filters in the literature are either not applicable for some problems or do not preserve unbiasedness in the presence of uncertainties, we have proposed a new robust low-order filter design method. An LPV formulation of the

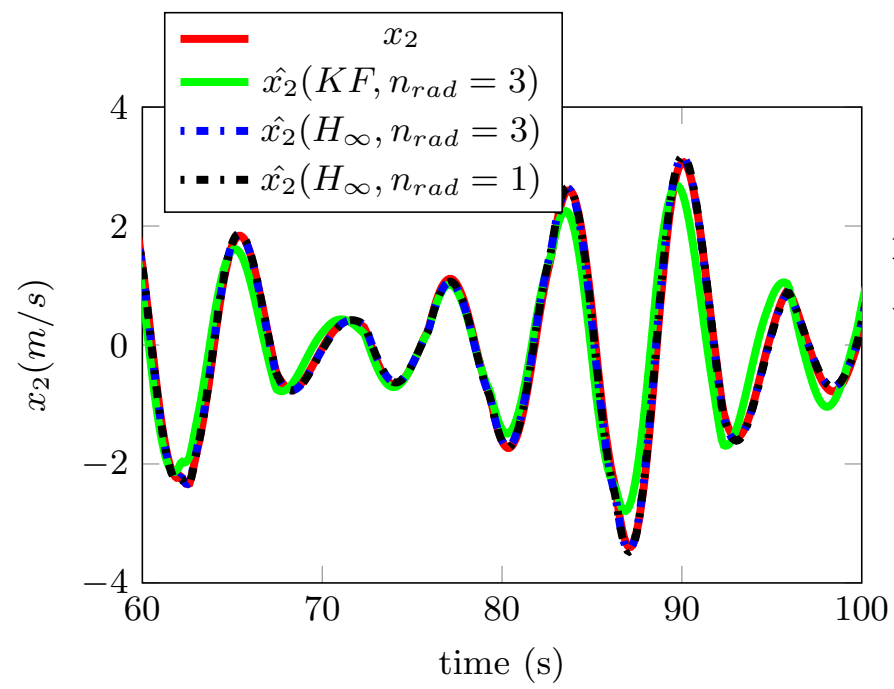

(a) velocity estimates

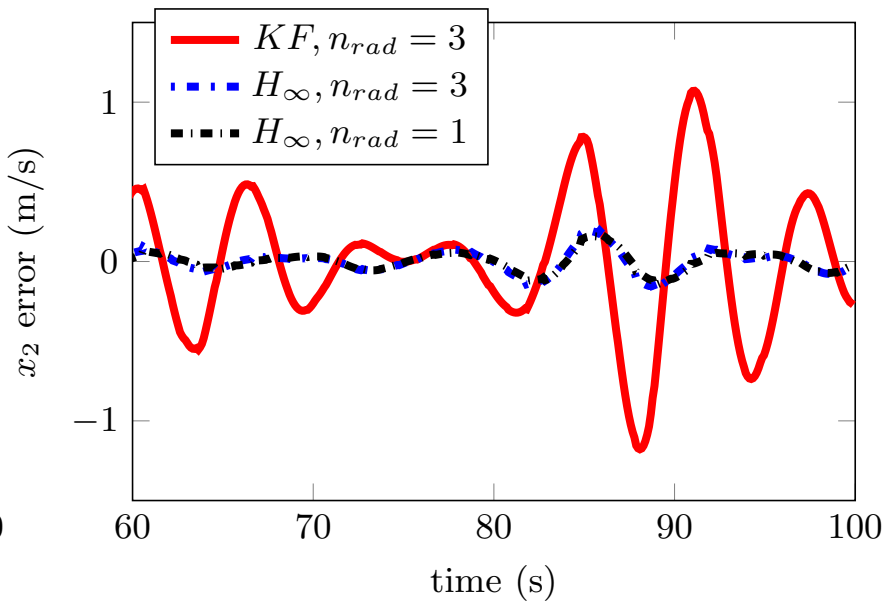

(b) errors in velocity estimates

Fig. 9. Velocity estimates with observers based on radiation subsystems of order $n_{\text {rad }}$ equal to 1 and 3 for the $H_{\infty}$ filter, and a Kalman Filter (KF) based on a $3^{\text {rd }}$ order radiation subsystem. 


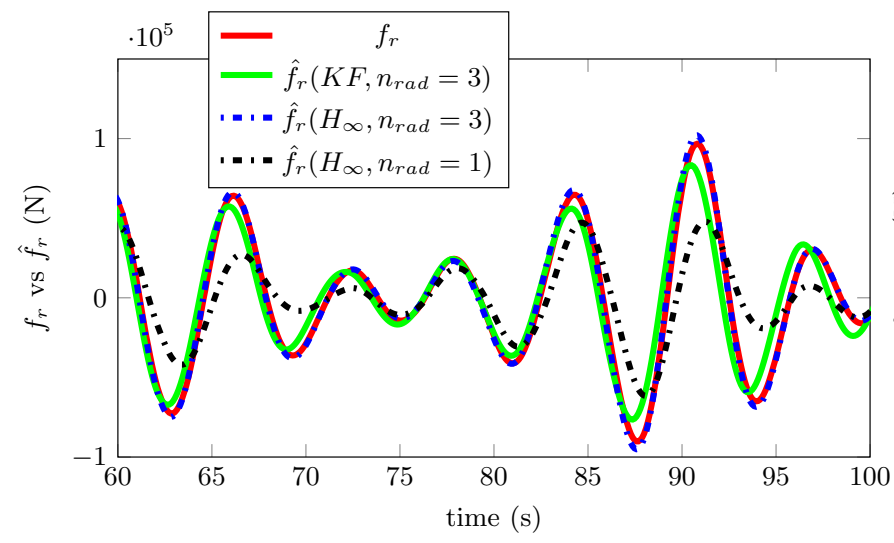

(a) radiation force estimates

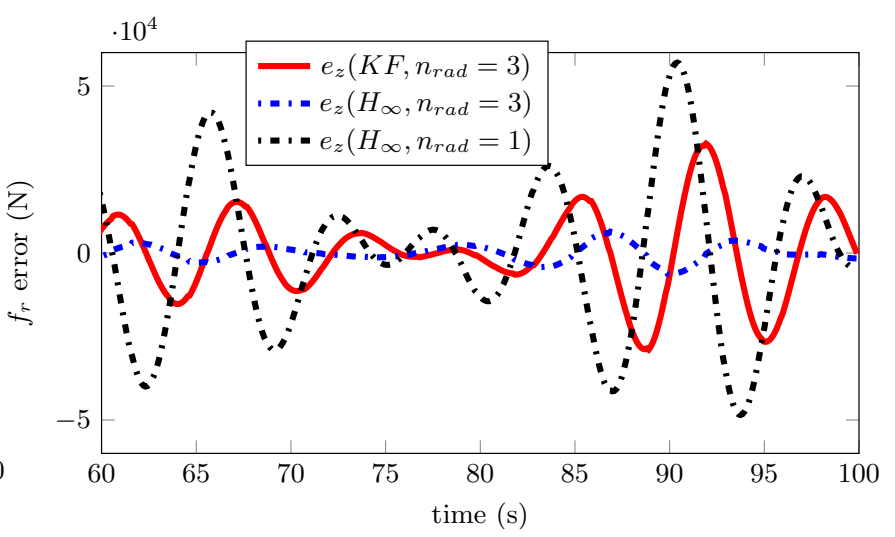

(b) errors in radiation force estimates

Fig. 10. Radiation force estimates with observers based on radiation subsystems of order $n_{\text {rad }}$ equal to 1 and 3 for the $H_{\infty}$ filter, and a Kalman Filter (KF) based on a $3^{\text {rd }}$ order radiation subsystem.

bilinear system has been used to design a filter that is based on a lower order model of the plant, but with closed-loop robust disturbance attenuation guarantees around the full-order model. Robustness against linear parameter uncertainties is explicitly considered in the synthesis. Although the optimal filter synthesis is a (non-convex) BMI problem, we have used an efficient LMI-based coordinate descent algorithm to find solutions locally. Example simulations in dynamic radiation force estimation of a wave energy converter have been used to show robustness of the $H_{\infty}$ filter under parametric and other model uncertainties by comparing the performance with an LTV Kalman filter.

\section{REFERENCES}

[1] P. M. Pardalos and V. Yatsenko, Eds., Optimization and Control of Bilinear Systems: Theory, Algorithms, and Applications. Springer Berlin, 2008, vol. 13.

[2] E. Abraham and E. C. Kerrigan, “Optimal active control and optimization of a wave energy converter," IEEE Transactions on Sustainable Energy, vol. 4, no. 2, pp. 324-332, 2013.

[3] C. E. de Souza and A. Trofino, "A linear matrix inequality approach to the design of robust $\mathrm{H}_{2}$ filters," in Advances in Linear Matrix Inequality Methods in Control, L. E. Ghaoui and S. I. Niculescu, Eds. Society for Industrial and Applied Mathematics, 2000, ch. 9, pp. 175-185.

[4] H. Li and M. Fu, "A linear matrix inequality approach to robust $\mathscr{H}_{\infty}$ filtering," IEEE Transactions on Signal Processing, vol. 45, no. 9, pp. 2338-2350, 1997.

[5] C. E. de Souza, U. Shaked, and M. Fu, "Robust $\mathscr{H}_{\infty}$ filtering for continuous time varying uncertain systems with deterministic input signals," IEEE Transactions on Signal Processing, vol. 43, no. 3, pp. 709-719, 1995.

[6] Z. Wang, Y. Liu, and X. Liu, “ $\mathscr{H}_{\infty}$ filtering for uncertain stochastic time-delay systems with sector-bounded nonlinearities,” Automatica, vol. 44, no. 5, pp. 1268-1277, 2008.

[7] L. E. Ghaoui and S. I. Niculescu, Advances in linear matrix inequality methods in control. Society for Industrial Mathematics, 2000, vol. 2.

[8] R. Rajamani, "Observers for Lipschitz nonlinear systems," IEEE Transactions on Automatic Control, vol. 43, no. 3, pp. 397-401, 1998.

[9] A. Alessandri, "Observer design for nonlinear systems by using input-to-state stability," in Decision and Control (CDC), 2004. 43rd IEEE Conference on, vol. 4. IEEE, 2004, pp. 3892-3897.

[10] M. Abbaszadeh and H. J. Marquez, "Robust $\mathscr{H}_{\infty}$ observer design for a class of nonlinear uncertain systems via convex optimization," in American Control Conference (ACC), 2007. IEEE, 2007, pp. 1699-1704.

[11] B. Song and J. K. Hedrick, "Nonlinear observer design for Lipschitz nonlinear systems," in American Control Conference (ACC), 2011. IEEE, 2011, pp. 2578-2583.

[12] B. Gérard, H. S. Ali, M. Zasadzinski, and M. Darouach, " $H_{\infty}$-filter for bilinear systems using LPV approach,” IEEE Transactions on Automatic Control, vol. 55, no. 7, pp. 1668-1674, 2010.

[13] H. S. Ali, M. Zasadzinski, H. Rafaralahy, and M. Darouach, "Robust $H_{\infty}$ reduced order filtering for uncertain bilinear systems," Automatica, vol. 42, no. 3, pp. $405-415,2006$. 
[14] M. Zasadzinski, H. S. Ali, and M. Darouach, "Robust reduced order unbiased filtering for uncertain systems," International Journal of Control, vol. 79, no. 2, pp. 93-106, 2006.

[15] I. J. Couchman, E. C. Kerrigan, and C. Böhm, "Model reduction of homogeneous-in-the-state bilinear systems with input constraints," Automatica, vol. 47, no. 4, pp. 761-768, 2011.

[16] A. C. Antoulas, Approximation of large-scale dynamical systems. Society for Industrial Mathematics, 2005, vol. 6.

[17] B. L. Jones and E. C. Kerrigan, "When is the discretization of a spatially distributed system good enough for control?" Automatica, vol. 46, no. 9, pp. $1462-1468,2010$

[18] K. M. Grigoriadis and J. T. W. Jr, "Reduced-order $H_{\infty}$ and $L_{2}-L_{\infty}$ filtering via linear matrix inequalities," IEEE Transactions on Aerospace and Electronic Systems, vol. 33, no. 4, pp. 1326-1338, 1997.

[19] S. Boyd, L. E. Ghaoui, E. Feron, and V. Balakrishnan, Linear matrix inequalities in system and control theory. Society for Industrial Mathematics, 1994, vol. 15 .

[20] H. K. Khalil, Nonlinear systems. Prentice Hall, 2002, vol. 3.

[21] J. S. Shamma, An Overview of LPV Systems. Springer, 2012, pp. 3-26.

[22] M. Henk, J. Richter-Gebert, and G. M. Ziegler, "16 basic properties of convex polytopes," Handbook of discrete and computational geometry, pp. 255-382, 2004.

[23] C. Scherer and S. Weiland, "Linear matrix inequalities in control," DISC Course Lecture Notes, 2000.

[24] S. P. Boyd and L. Vandenberghe, Convex optimization. Cambridge Univ Pr, 2004.

[25] K. Grigoriadis and E. Beran, "Alternating projection algorithms for linear matrix inequalities problems with rank constraints," in Advances in Linear Matrix Inequality Methods in Control, L. E. Ghaoui and S. I. Niculescu, Eds. Society for Industrial Mathematics, 2000, ch. 13, pp. $251-267$.

[26] P. Gahinet, P. Apkarian, and M. Chilali, "Affine parameter-dependent lyapunov functions and real parametric uncertainty," IEEE Transactions on Automatic Control, vol. 41, no. 3, pp. 436-442, 1996.

[27] R. Shorten, F. Wirth, O. Mason, K. Wulff, and C. King, "Stability criteria for switched and hybrid systems," SIAM review, vol. 49, no. 4, pp. 545-592, 2007.

[28] V. Blondel and J. N. Tsitsiklis, "Np-hardness of some linear control design problems," SIAM Journal on Control and Optimization, vol. 35, no. 6, pp. 2118-2127, 1997.

[29] T. D. Quoc, S. Gumussoy, W. Michiels, and M. Diehl, "Combining convex-concave decompositions and linearization approaches for solving BMIs, with application to static output feedback," IEEE Transactions on Automatic Control, vol. 57, no. 6, pp. 1377-1390, 2012.

[30] K.-C. Goh, M. G. Safonov, and G. P. Papavassilopoulos, "Global optimization for the biaffine matrix inequality problem," Journal of global optimization, vol. 7, no. 4, pp. 365-380, 1995.

[31] C. W. J. Hols and C. W. Scherer, "A sum-of-squares approach to fixed-order $H_{\infty}$-synthesis," Positive Polynomials in Control, pp. 580-580, 2005.

[32] D. Henrion and J.-B. Lasserre, "Convergent relaxations of polynomial matrix inequalities and static output feedback," IEEE Transactions on Automatic Control, vol. 51, no. 2, pp. 192-202, 2006.

[33] S. Kanev, C. Scherer, M. Verhaegen, and B. D. Schutter, "Robust output-feedback controller design via local bmi optimization," Automatica, vol. 40, no. 7, pp. 1115-1127, 2004.

[34] E. Simon, P. Rodriguez-Ayerbe, C. Stoica, D. Dumur, and V. Wertz, "LMI-based coordinate descent method for solving BMIs in control design: A short survey \& two improvements," in Proceedings of the 18th IFAC World Congress, 2011.

[35] M. Kocvara and M. Stingl, PENBMI User's Guide (Version 2.1), PENOPT GbR, www.penopt.com, February 2012.

[36] M. Kočvara and M. Stingl, "PENNON: A code for convex nonlinear and semidefinite programming," Optimization methods and software, vol. 18, no. 3, pp. 317-333, 2003.

[37] M. K. F. Leibfritz, M. Stingl, and D. Henrion, “A nonlinear sdp algorithm for static output feedback problems in compleib," in Proceedings of the 16th IFAC World Congress on Automatic Control, Prague, 2005.

[38] F. Leibfritz, "COMPleib: COnstrained Matrix optimization Problem library," 2006.

[39] C. Scherer, P. Gahinet, and M. Chilali, "Multiobjective output-feedback control via lmi optimization," IEEE Transactions on Automatic Control, vol. 42, no. 7, pp. 896-911, 1997.

[40] T. Iwasaki, “The dual iteration for fixed-order control," IEEE Transactions on Automatic Control, vol. 44, no. 4, pp. 783-788, 1999.

[41] J. Lofberg, "YALMIP: A toolbox for modeling and optimization in Matab," in Proc. 2004 IEEE International Symposium on Computer Aided Control Systems Design. IEEE, 2004, pp. 284-289. 
This is the author's version of an article that has been published in this journal. Changes were made to this version by the publisher prior to publication.

The final version of record is available at http://dx.doi.org/10.1109/TSP.2014.2385656

[42] E. Simon and V. Wertz, "Improvement of the LMI change of variables for multi-objective control design problems," Optimal Control Applications and Methods, 2012. [Online]. Available: http://dx.doi.org/10.1002/oca.2040

[43] D. Simon, Optimal state estimation: Kalman, $H_{\infty}$, and nonlinear approaches. Wiley-Interscience, 2006.

[44] M. Green and D. J. N. Limebeer, Linear robust control. Prentice Hall, 1994.

[45] K. M. Nagpal and P. P. Khargonekar, "Filtering and smoothing in an $H_{\infty}$ setting," IEEE Transactions on Automatic Control, vol. 36, no. 2, pp. 152-166, 1991.

[46] K. C. Toh, M. J. Todd, and R. H. Tütüncü, "SDPT3—a Matab software package for semidefinite programming, version 1.3," Optimization Methods and Software, vol. 11, no. 1-4, pp. 545-581, 1999.

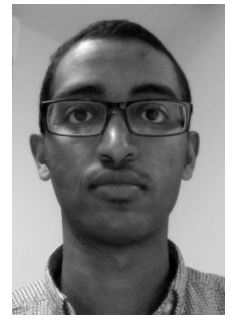

Edo Abraham received the M.Eng and PhD degrees from Imperial College London, U.K., in 2008 and 2013, respectively.

He is currently a Research Associate in the Environmental and Water Resources Engineering Group, Imperial College London. His research interests focus on the development of tailored linear solvers, optimal control, estimation and optimization tools for smart water distribution systems.

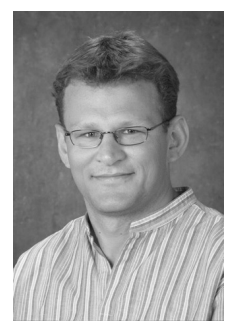

Eric C. Kerrigan (S'94-M'02) received a PhD from the University of Cambridge in 2001 and has been with Imperial College London since 2006. His research is focused on the development of efficient numerical methods and embedded computing architectures for solving advanced optimization, control and estimation problems in real-time. His work is applied to a variety of problems in the design of cyber-physical and computing systems that arise in aerospace and renewable energy applications. He is on the IEEE Control Systems Society Conference Editorial Board and is an associate editor of the IEEE Transactions on Control Systems Technology, Control Engineering Practice, and Optimal Control Applications and Methods. 